\title{
A necessary and sufficient condition for sequences to be minimal completely monotonic
}

\author{
Xi-Feng Wang ${ }^{1 *}$, Mourad E.H. Ismail ${ }^{2,3}$, Necdet Batir ${ }^{4}$ and S. Guo ${ }^{1}$ (D)
}

Dedicated to Professor Hari M. Srivastava on the occasion of his eightieth birthday.

\section{"Correspondence:}

wang-xifeng@hotmail.com;

5390@zut.edu.cn

1 Department of Applied

Mathematics, Zhongyuan University

of Technology, Zhengzhou, Henan

450007, People's Republic of China

Full list of author information is

available at the end of the article

\begin{abstract}
In this article, we present a necessary and sufficient condition under which sequences are minimal completely monotonic.
\end{abstract}

MSC: Primary 44A60; secondary 44A10

Keywords: Completely monotonic sequence; Completely monotonic function; Minimal completely monotonic sequence

\section{Introduction and the main results}

We first recall some definitions and basic results on completely monotonic sequences and minimal completely monotonic sequences.

Definition 1 ([20]) A sequence $\left\{\mu_{n}\right\}_{n=0}^{\infty}$ is called completely monotonic if

$$
(-1)^{k} \Delta^{k} \mu_{n} \geq 0, \quad n, k \in \mathbb{N}_{0}:=\{0\} \cup \mathbb{N}
$$

where

$$
\Delta^{0} \mu_{n}=\mu_{n}
$$

and

$$
\Delta^{k+1} \mu_{n}=\Delta^{k} \mu_{n+1}-\Delta^{k} \mu_{n}
$$

Here in Definition 1, and throughout the paper, $\mathbb{N}$ is the set of all positive integers and $\mathbb{N}_{0}$ is the set of all nonnegative integers.

Widder [25] defined a sub-class of the class of completely monotonic sequences.

(c) The Author(s) 2020. This article is licensed under a Creative Commons Attribution 4.0 International License, which permits use, sharing, adaptation, distribution and reproduction in any medium or format, as long as you give appropriate credit to the original author(s) and the source, provide a link to the Creative Commons licence, and indicate if changes were made. The images or other third party material in this article are included in the article's Creative Commons licence, unless indicated otherwise in a credit line 
Definition 2 A sequence $\left\{\mu_{n}\right\}_{n=0}^{\infty}$ is called minimal completely monotonic if it is completely monotonic and if it will not be completely monotonic when $\mu_{0}$ is replaced by a number less than $\mu_{0}$.

Regarding the relationships between completely monotonic sequences and minimal completely monotonic sequences, in [6] the author proved that if the sequence $\left\{\mu_{n}\right\}_{n=0}^{\infty}$ is completely monotonic, then:

(1) for any $m \in \mathbb{N}$, the sequence $\left\{\mu_{n}\right\}_{n=m}^{\infty}$ is minimal completely monotonic, and

(2) there exists one (then only one) number $\mu_{0}^{*}$ such that the sequence

$$
\left\{\mu_{0}^{*}, \mu_{1}, \mu_{2}, \ldots\right\}
$$

is minimal completely monotonic.

Please note that the complete monotonicity of the sequence $\left\{\mu_{n}\right\}_{n=1}^{\infty}$ cannot guarantee that there exists a number $\mu_{0}^{*}$ such that the sequence

$$
\left\{\mu_{0}^{*}, \mu_{1}, \mu_{2}, \ldots\right\}
$$

is completely monotonic. In fact, if the sequence (4) is completely monotonic, then the sequence $\left\{\mu_{n}\right\}_{n=1}^{\infty}$ should be minimal completely monotonic.

In [18] the authors showed that if the sequence $\left\{\mu_{n}\right\}_{n=0}^{\infty}$ is completely monotonic, then, for any $m \in \mathbb{N}_{0}$, the series

$$
\sum_{j=0}^{\infty}(-1)^{j} \Delta^{j} \mu_{m+1}
$$

converges and

$$
\mu_{m} \geq \sum_{j=0}^{\infty}(-1)^{j} \Delta^{j} \mu_{m+1} .
$$

We also recall the following definition.

Definition 3 ([4]) A function $f$ is said to be completely monotonic on an interval $I$, if $f \in C(I)$, has derivatives of all orders on $I^{o}$ (the interior of $I$ ) and for all $n \in \mathbb{N}_{0}$

$$
(-1)^{n} f^{(n)}(x) \geq 0, \quad x \in I^{o}
$$

Here in Definition $3 C(I)$ is the space of all continuous functions on the interval $I$. The class of all completely monotonic functions on the interval $I$ is denoted by $C M(I)$.

There is rich literature on completely monotonic functions and sequences, and their applications. For more recent works, see, for example, [1-3, 5-19, 21-24].

For sequences to be interpolated by completely monotonic functions, Widder [25] proved that there exists a function

$$
f \in C M[0, \infty)
$$


such that

$$
f(n)=\mu_{n}, \quad n \in \mathbb{N}_{0}
$$

if and only if the sequence $\left\{\mu_{n}\right\}_{n=0}^{\infty}$ is minimal completely monotonic. From this we see that the condition of minimal complete monotonicity is critical for a sequence $\left\{\mu_{n}\right\}_{n=0}^{\infty}$ to be interpolated by a completely monotonic function on the interval $[0, \infty)$.

In this article, we shall further investigate on minimal completely monotonic sequences. The main results of this article are as follows.

Theorem 4 Suppose that the sequence $\left\{\mu_{n}\right\}_{n=1}^{\infty}$ is completely monotonic and that the series

$$
\sum_{j=0}^{\infty}(-1)^{j} \Delta^{j} \mu_{1}
$$

converges. Let

$$
\mu_{0}^{*}:=\sum_{j=0}^{\infty}(-1)^{j} \Delta^{j} \mu_{1} .
$$

Then the sequence

$$
\left\{\mu_{0}^{*}, \mu_{1}, \mu_{2}, \mu_{3}, \ldots\right\}
$$

is minimal completely monotonic.

Remark 5 It should be noted that the condition: "the series

$$
\sum_{j=0}^{\infty}(-1)^{j} \Delta^{j} \mu_{1}
$$

converges" in Theorem 4 cannot be dropped since the complete monotonicity of the sequence $\left\{\mu_{n}\right\}_{n=1}^{\infty}$ cannot guarantee the convergence of the series

$$
\sum_{j=0}^{\infty}(-1)^{j} \Delta^{j} \mu_{1} .
$$

For example, let

$$
\mu_{n}=\frac{1}{n}, \quad n \in \mathbb{N}
$$

We can verify that the sequence $\left\{\mu_{n}\right\}_{n=1}^{\infty}$ is completely monotonic and that

$$
\Delta^{j} \mu_{1}=\frac{(-1)^{j}}{j+1}
$$


Hence

$$
\sum_{j=0}^{\infty}(-1)^{j} \Delta^{j} \mu_{1}=\sum_{j=0}^{\infty} \frac{1}{j+1}
$$

which is divergent.

Theorem 6 Suppose that the sequence $\left\{\mu_{n}\right\}_{n=0}^{\infty}$ is minimal completely monotonic. Then the series

$$
\sum_{j=0}^{\infty}(-1)^{j} \Delta^{j} \mu_{1}
$$

converges and

$$
\mu_{0}=\sum_{j=0}^{\infty}(-1)^{j} \Delta^{j} \mu_{1} .
$$

Theorem 7 A necessary and sufficient condition for the sequence $\left\{\mu_{n}\right\}_{n=0}^{\infty}$ to be minimal completely monotonic is that the sequence $\left\{\mu_{n}\right\}_{n=1}^{\infty}$ is completely monotonic, the series

$$
\sum_{j=0}^{\infty}(-1)^{j} \Delta^{j} \mu_{1}
$$

converges, and

$$
\mu_{0}=\sum_{j=0}^{\infty}(-1)^{j} \Delta^{j} \mu_{1} .
$$

\section{Proof of the main results}

Now we are in a position to prove the main results.

Proof of Theorem 4 By Theorem 11 in [18], we see that the sequence

$$
\left\{\mu_{0}^{*}, \mu_{1}, \mu_{2}, \mu_{3}, \ldots\right\}
$$

is completely monotonic. By Theorem 9 in [18], if a sequence

$$
\left\{\mu_{0}, \mu_{1}, \mu_{2}, \mu_{3}, \ldots\right\}
$$

is completely monotonic, then

$$
\mu_{0} \geq \sum_{j=0}^{\infty}(-1)^{j} \Delta^{j} \mu_{1}=\mu_{0}^{*}
$$

Hence by the definition of minimal completely monotonic sequence, we know that the sequence

$$
\left\{\mu_{0}^{*}, \mu_{1}, \mu_{2}, \mu_{3}, \ldots\right\}
$$


is minimal completely monotonic. The proof of Theorem 4 is completed.

Proof of Theorem 6 Since the sequence

$$
\left\{\mu_{0}, \mu_{1}, \mu_{2}, \mu_{3}, \ldots\right\}
$$

is completely monotonic, by Theorem 9 in [18], the series

$$
\sum_{j=0}^{\infty}(-1)^{j} \Delta^{j} \mu_{1}
$$

converges and

$$
\mu_{0} \geq \sum_{j=0}^{\infty}(-1)^{j} \Delta^{j} \mu_{1}
$$

By Theorem 11 in [18], we see that the sequence

$$
\left\{\sum_{j=0}^{\infty}(-1)^{j} \Delta^{j} \mu_{1}, \mu_{1}, \mu_{2}, \mu_{3}, \ldots\right\}
$$

is completely monotonic. Since the completely monotonic sequence

$$
\left\{\mu_{0}, \mu_{1}, \mu_{2}, \mu_{3}, \ldots\right\}
$$

is minimal, we have

$$
\mu_{0} \leq \sum_{j=0}^{\infty}(-1)^{j} \Delta^{j} \mu_{1}
$$

From (21) and (24), we get our conclusion. The proof of Theorem 6 is completed.

Proof of Theorem 7 By the definition of completely monotonic sequence, Theorem 9 in [18] and Theorem 6, we know that the condition is necessary. By Theorem 4, we see that the condition is sufficient. The proof of Theorem 7 is thus completed.

\section{Conclusion}

In this paper, we investigated properties of completely monotonic sequences. We have proved a necessary condition for a sequence to be a minimal completely monotonic sequence. We also have presented a necessary and sufficient condition under which sequences are minimal completely monotonic.

\section{Acknowledgements}

The authors thank the editor and the reviewers for their valuable suggestions and comments which have improved the manuscript significantly. 
Availability of data and materials

Not applicable.

\section{Competing interests}

The authors declare that they have no competing interests.

\section{Authors' contributions}

All the authors contributed to the writing of the present article. They also read and approved the final manuscript.

\section{Author details}

'Department of Applied Mathematics, Zhongyuan University of Technology, Zhengzhou, Henan 450007, People's Republic of China. ${ }^{2}$ Department of Mathematics, King Saud University, Riyadh, Saudi Arabia. ${ }^{3}$ Department of Mathematics, University of Central Florida, Orlando, Florida 32816, USA. ${ }^{4}$ Department of Mathematics, Nevşehir University, Nevşehir TR-50300, Turkey.

\section{Publisher's Note}

Springer Nature remains neutral with regard to jurisdictional claims in published maps and institutional affiliations.

Received: 1 May 2020 Accepted: 11 October 2020 Published online: 26 November 2020

\section{References}

1. Alzer, H., Batir, N.: Monotonicity properties of the gamma function. Appl. Math. Lett. 20, 778-781 (2007)

2. Alzer, H., Berg, C., Koumandos, S.: On a conjecture of Clark and Ismail. J. Approx. Theory 134, 102-113 (2005)

3. Batir, N.: On some properties of the gamma function. Expo. Math. 26, 187-196 (2008)

4. Bernstein, S.: Sur la définition et les propriétés des fonctions analytiques d'une variable réelle. Math. Ann. 75, 449-468 (1914)

5. Guo, S.: Logarithmically completely monotonic functions and applications. Appl. Math. Comput. 221, $169-176$ (2013)

6. Guo, S.: Some properties of completely monotonic sequences and related interpolation. Appl. Math. Comput. 219, 4958-4962 (2013)

7. Guo, S.: A class of logarithmically completely monotonic functions and their applications. J. Appl. Math. 2014, 757462 (2014)

8. Guo, S.: Some conditions for a class of functions to be completely monotonic. J. Inequal. Appl. 2015, 11 (2015)

9. Guo, S.: On completely monotonic and related functions. Filomat 30, 2083-2090 (2016)

10. Guo, S.: Some properties of functions related to completely monotonic functions. Filomat 31, 247-254 (2017)

11. Guo, S., Laforgia, A., Batir, N., Luo, Q.-M.: Completely monotonic and related functions: their applications. J. Appl. Math. $2014,768516(2014)$

12. Guo, S., Qi, F.: A class of logarithmically completely monotonic functions associated with the gamma function. J. Comput. Appl. Math. 224, 127-132 (2009)

13. Guo, S., Qi, F., Srivastava, H.M.: Necessary and sufficient conditions for two classes of functions to be logarithmically completely monotonic. Integral Transforms Spec. Funct. 18, 819-826 (2007)

14. Guo, S., Qi, F., Srivastava, H.M.: Supplements to a class of logarithmically completely monotonic functions associated with the gamma function. Appl. Math. Comput. 197, 768-774 (2008)

15. Guo, S., Qi, F., Srivastava, H.M.: A class of logarithmically completely monotonic functions related to the gamma function with applications. Integral Transforms Spec. Funct. 23, 557-566 (2012)

16. Guo, S., Srivastava, H.M.: A class of logarithmically completely monotonic functions. Appl. Math. Lett. 21, 1134-1141 (2008)

17. Guo, S., Srivastava, H.M.: A certain function class related to the class of logarithmically completely monotonic functions. Math. Comput. Model. 49, 2073-2079 (2009)

18. Guo, S., Srivastava, H.M., Batir, N.: A certain class of completely monotonic sequences. Adv. Differ. Equ. 2013, 294 (2013)

19. Guo, S., Srivastava, H.M., Cheung, W.S.: Some properties of functions related to certain classes of completely conotonic functions and logarithmically completely conotonic functions. Filomat 28, 821-828 (2014)

20. Hausdorff, F.: Summationsmethoden und momentfolgen I. Math. Z. 9, 74-109 (1921)

21. Qi, F., Guo, S., Guo, B.-N.: Complete monotonicity of some functions involving polygamma functions. J. Comput. Appl. Math. 233, 2149-2160 (2010)

22. Salem, A.: A completely monotonic function involving q-gamma and q-digamma functions. J. Approx. Theory 164 971-980 (2012)

23. Sevli, H., Batir, N.: Complete monotonicity results for some functions involving the gamma and polygamma functions. Math. Comput. Model. 53,1771-1775 (2011)

24. Srivastava, H.M., Guo, S., Qi, F.: Some properties of a class of functions related to completely monotonic functions. Comput. Math. Appl. 64, 1649-1654 (2012)

25. Widder, D.V.: Necessary and sufficient conditions for the representation of a function as a Laplace integral. Trans. Am. Math. Soc. 33, 851-892 (1931) 